\title{
Neural Responses to Unattended Products Predict Later Consumer Choices
}

\author{
Anita Tusche, ${ }^{1,2}$ Stefan Bode, ${ }^{1,2,3}$ and John-Dylan Haynes ${ }^{1,2}$ \\ ${ }^{1}$ Bernstein Center for Computational Neuroscience Berlin, Charité-Universitätsmedizin Berlin, 10115 Berlin, Germany, ${ }^{2}$ Max-Planck-Institute for Human \\ Cognitive and Brain Sciences, 04103 Leipzig, Germany, and ${ }^{3}$ Department of Neurology, Otto-von-Guericke University, 39120 Magdeburg, Germany
}

Imagine you are standing at a street with heavy traffic watching someone on the other side of the road. Do you think your brain is implicitly registering your willingness to buy any of the cars passing by outside your focus of attention? To address this question, we measured brain responses to consumer products (cars) in two experimental groups using functional magnetic resonance imaging. Participants in the first group (high attention) were instructed to closely attend to the products and to rate their attractiveness. Participants in the second group (low attention) were distracted from products and their attention was directed elsewhere. After scanning, participants were asked to state their willingness to buy each product. During the acquisition of neural data, participants were not aware that consumer choices regarding these cars would subsequently be required. Multivariate decoding was then applied to assess the choice-related predictive information encoded in the brain during product exposure in both conditions. Distributed activation patterns in the insula and the medial prefrontal cortex were found to reliably encode subsequent choices in both the high and the low attention group. Importantly, consumer choices could be predicted equally well in the low attention as in the high attention group. This suggests that neural evaluation of products and associated choice-related processing does not necessarily depend on attentional processing of available items. Overall, the present findings emphasize the potential of implicit, automatic processes in guiding even important and complex decisions.

\section{Introduction}

Brain responses obtained during active evaluation of products and explicit deliberation about purchases have been found to predict consumer choices (Knutson et al., 2007). Interestingly, some evidence suggests that automatic brain processes might guide human judgments and choices, even in the absence of explicit deliberation and attention to the choice task. Brain responses were shown to engage automatically in assessing facial attractiveness and preferences even when such judgments were not part of the designated task (O'Doherty et al., 2003; Kim et al., 2007). Likewise, brain activation was reported to reflect preferences when participants evaluate stimuli with respect to other, non-preference-related aspects (Lebreton et al., 2009). However, the precise role of stimulus-related attention in mediating such automatic valuation processes remains unclear. On the one hand, sensory responses to unattended stimuli have been shown be strongly reduced (Rees et al., 1997; Martínez et al., 1999; Kastner and Ungerleider, 2000). On the other hand, spatially unattended stimuli have been reported to undergo substantial category-

Received Jan. 6, 2010; revised March 16, 2010; accepted April 8, 2010.

This work was funded by the Max Planck Society, the German Research Foundation, and the Bernstein Computational Neuroscience Program of the German Federal Ministry of Education and Research. We thank Christian Kalberlah, Chun Siong Soon, and Carsten Bogler for their contributions to this project and Thorsten Kahnt for comments on an earlier version of the manuscript.

Correspondence should be addressed to John-Dylan Haynes or Anita Tusche, Charité-Universitätsmedizin Berlin Bernstein Center for Computational Neuroscience, Haus 6, Philippstrasse 13, 10115 Berlin, Germany. E-mail: haynes@bccn-berlin.de. or anita.tusche@bccn-berlin.de.

DOI:10.1523/JNEUROSCI.0064-10.2010

Copyright $\odot 2010$ the authors $\quad 0270-6474 / 10 / 308024-08 \$ 15.00 / 0$ selective processing (Peelen et al., 2009). To date, no study has directly compared neural responses to attended versus unattended products, and the impact of spatial attention on the prediction of economic decisions. Here, we investigated whether brain responses predict consumer choices even when products are entirely task-irrelevant and presented outside the focus of attention.

To examine the role of attention in the prediction of product choices from brain activity, we performed an experiment with two different groups of male participants. In each trial, participants were presented with an image of a car while their brain responses were measured using functional magnetic resonance imaging (fMRI). Participants in group 1 (high attention) were instructed to actively evaluate and rate the attractiveness of each particular car after its presentation (Fig. 1A). Hence, functional brain responses were acquired while products were task-relevant and in the focus of attention. In contrast, participants in group 2 (low attention), were engaged in a demanding visual fixation task while task-irrelevant images of cars were passively presented outside the focus of attention in the background of the screen (Fig. 1B). After the scanning session, participants from both groups were instructed to realistically picture themselves in a consumer setting where they had to decide on a new car. For each of the previously presented products, participants were then asked to state whether they would like to purchase this car or not (Fig. 1C). During scanning, participants from both groups were unaware that they would later be asked about their potential purchases. Multivariate pattern classification (Haxby et al., 2001; Kriegeskorte et al., 2006; Norman et al., 2006; Haynes et al., 2007) was then applied 
Scanning session

\section{A Group 1 (high attention)}

Active evaluation of products ("How much do you like this car?")

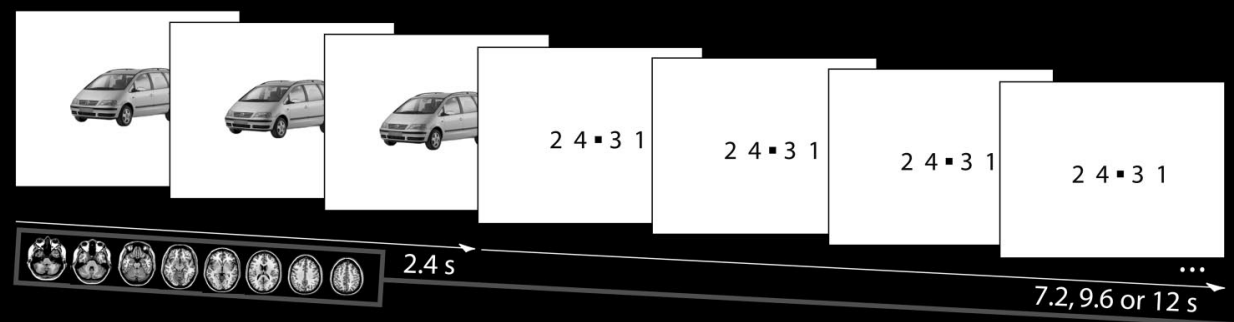

B Group 2 (low attention) Passive exposure to products ("Perform the fixation task!")

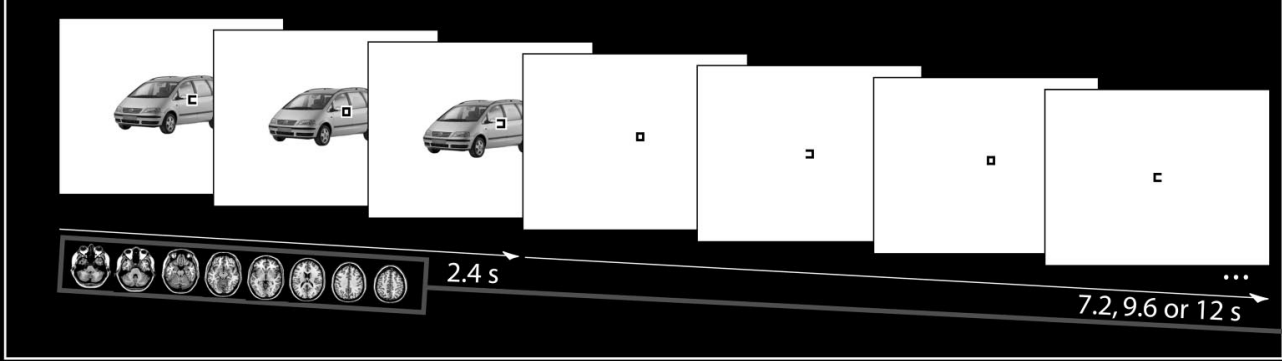

After scanning session

\section{Questionnaire}

"Would you buy this car?"

Predict choice
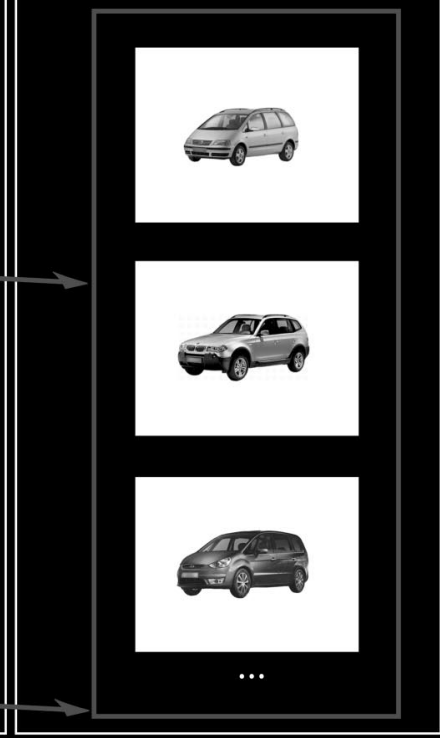

Figure 1. Experimental paradigms. A, In each trial, participants in group 1 (high attention) were presented with images of single cars for 2.4 s followed by a randomized response-button mapping screen (displayed for a variable duration of 7.2-12 s). Participants were instructed to closely attend to products and to actively evaluate the attractiveness of the particular car on a four-point scale by pressing the corresponding response button. $\boldsymbol{B}$, Participants in group 2 (low attention) were asked to perform a demanding fixation task. They responded to the opening of a centrally presented fixation square (every $800 \mathrm{~ms}$ ) with a corresponding left- or right-hand button press. The timing parameters applied in group 1 were retained. Every $7.6-12 \mathrm{~s}$, a car was passively presented on the background of the screen for $2.4 \mathrm{~s}$ while the fixation task continued. C, After the scanning session, participants from both groups were instructed to realistically picture themselves in a consumer setting where they had to decide upon a new car. Participants were then asked to state their willingness to buy each product. During scanning, participants of both groups were not informed that such a consumer choice would subsequently be required.

to the brain responses obtained during product exposure to predict their stated product choices.

The present study examined the impact of attention on neural predictors of economic decision making. More precisely, we investigated the predictability of consumer choices from brain responses for unattended products. A successful prediction would indicate that product valuation and associated choice-related processing can take place automatically-even with attention diverted from products.

\section{Materials and Methods}

Participants and stimuli. Group 1 consisted of 17 participants (aged between 24 and 32 years) and group 2 of 15 participants (aged between 22 and 27 years). Participants were healthy male volunteers, had normal or corrected-to-normal vision, were right-handed and stated that they were interested in cars before the experiment. The sample was limited to males interested in cars to ensure that participants were familiar with and possess stable mental representations about a wide range of items from the product group. Both paradigms were approved by the local ethics committee. All participants were paid $€ 12$ to take part and all gave written informed consent.

In both fMRI paradigms, monochrome images of 10 real life cars were used as stimuli. The selection of cars was based on a behavioral pretest with an independent sample of participants in a way that maximized variability in ratings between participants. All pictures were obtained from the Internet and were normalized with regard to size and contrast. During both fMRI paradigms, images were centrally presented against a white background using MATLAB 7.0 (The MathWorks) and the Cogent toolbox (http://www.vislab.ucl.ac.uk/Cogent). Data from four participants (in group 1 and 2) as well as data for one car (group 1) and two cars (group 2) was excluded because of missing variance in consumer choices.
Experimental paradigms. During each trial of both event-related fMRI paradigms, participants were presented with a single image of a car for 2.4 s. Participants in group 1 (high attention) were instructed to judge the subjective attractiveness of the specific car on a four-point-scale via a button-press ( 1 = "dislike it a lot," $2=$ "dislike it a little," $3=$ "like it a little," 4 = "like it a lot"). The mapping of buttons to attractiveness values was randomized on a trial-by-trial basis to avoid motor preparation during product exposure. Thus, each presentation of a car was followed by a randomized response-mapping screen, which was presented for 7.2-12 s. Responses were given using the index and middle fingers of both hands operating separate button boxes. Participants in group 2 (low attention) were instructed to attend to a black square presented centrally on a white screen. Every $800 \mathrm{~ms}$, the square opened to either the left or the right side. Participants had to respond to each opening with a matching left or right button press using the index fingers of both hands. After a pseudo-randomized duration of 7.2-12 s, a single image of a car was presented for $2.4 \mathrm{~s}$ in the background of the screen while the fixation task continued. Thus, the visual presentation of cars in group 2 mirrored that of group 1 but differed in the direction of attention that was diverted from products. Participants in group 2 were explicitly instructed to maximize performance on the fixation task throughout the entire experiment.

For both paradigms, scanning was performed in a single measurement session during which seven independent runs were acquired. The runs were separated by breaks of $\sim 1$ min during which no scanning data were obtained. Within a run, each of the 10 cars (see stimuli section above) was presented three times, resulting in a total number of 30 trials per run. For each run, the presentation order of cars was pseudo-randomized such that the same product was never shown in two consecutive trials. Pseudorandomized durations between presentations of cars varied between 7.2 to $12 \mathrm{~s}$, meaning that each car was combined with different interstimulus 
intervals equally often. This procedure ensured that the onset time of the next stimulus was unpredictable and event-related brain responses were clearly separable between trials.

Subsequent to the scanning sessions in both groups, participants were given a questionnaire with the question "Would you buy this car?" and the response options "No/Not sure/Yes." Importantly, during the acquisition of brain responses participants were unaware that it would be necessary to make such a choice later on. In addition to consumer choices, participants in group 2 had to explicitly judge the attractiveness of each particular car on a four-point scale. Moreover, for both groups familiarity ratings were obtained for all of the products presented. A between-subject design was chosen to ensure that participants in the "low attention" condition were completely unaware of the task-relevance of the products presented.

Image acquisition. For both groups, functional imaging was performed on a 3-Tesla Siemens TRIO scanner with a standard head coil. T2*weighted functional images were obtained using an echoplanar imaging (EPI) sequence [repetition time $(\mathrm{TR})=2.4 \mathrm{~s}$, echo time $=30 \mathrm{~ms}]$. For each run, 152 EPI volumes were collected (36 ascending axial slices per volume, slice thickness $2 \mathrm{~mm}$, in-plane resolution $3 \mathrm{~mm} \times 3 \mathrm{~mm}, 1 \mathrm{~mm}$ interslice gap, matrix size $64 \times 64$ ). The whole session consisted of seven runs. Due to technical problems, only five runs were acquired for two participants in group 2.

Data analysis. Data from both groups were analyzed in a similar manner. In a first step, the acquired volumes were slice timed and realigned. Preprocessed data were then analyzed using a general linear model (GLM) (Friston et al., 1994) as implemented in SPM2 (http://www.fil. ion.ucl.ac.uk/spm). For every run, parameter estimates for "purchase" and "no purchase" choices were estimated, based on stated consumer choices obtained after the scanning session. Additionally, biometric pulse data acquired during the scanning were included as coregressors of no interest to account for pulse artifacts. Four regressors were created by using the integral of the pulse curve of four successive time bins (TR/4= $0.6 \mathrm{~s}$ ) during the product presentation phase.

In the second step, multivariate pattern classification using a support vector machine (SVM) was applied to the parameter estimates of the consumer choices (Cox and Savoy, 2003; Mitchell et al., 2004; Kamitani and Tong, 2005; Haynes and Rees, 2006). To realize the classification a standard radial basis function kernel as implemented in LIBSVM (http:// www.csie.ntu.edu.tw/ cjlin/libsvm) was used. In contrast to a classifier restricted to linear effects, this approach allowed interactions between features and nonlinear functions to drive the prediction of subsequent choices. A comparative figure illustrating prediction accuracies obtained with a linear classification compared with our nonlinear approach is provided in supplemental Figure 1 (available at www.jneurosci.org as supplemental material).

Multivariate pattern classification techniques take advantage of information contained in multiple voxels distributed across space. They allow investigating whether spatial patterns of brain activation contain stable information about different experimental conditions (e.g., purchase vs no purchase). To achieve best predictive accuracy, the classifier weights the contributions of the different voxels optimally. In our case, some voxels within a searchlight cluster were weighted positively and others negatively by the classification algorithm. Moreover, the nonlinear classifier also takes their interactions into account.

To ensure an unbiased analysis of the neural activation patterns throughout the whole brain, a "searchlight" approach was used (Kriegeskorte et al., 2006; Haynes et al., 2007). Given that this approach does not depend on a priori assumptions about informative brain regions or prior voxel selection, the problem of circular analysis (or "double dipping") can be avoided (Kriegeskorte et al., 2009). For each participant, a sphere with a radius of 4 voxels was created around every voxel $v_{\mathrm{i}}$ of the measured volume. For each sphere, we investigated whether the local pattern of activation during product exposure predicted the willingness to buy that was stated after scanning (purchase vs no purchase). It should be noted that this analysis is predictive because it uses brain activity during exposure to predict purchase ratings obtained after scanning.

For every run, parameter estimates from the GLM were extracted for each of the $N$ voxels in the sphere around voxel $v_{\mathrm{i}}$ and transformed in an
$\mathrm{N}$-dimensional pattern vector. For each run, two pattern vectors were separately created for the purchase and no purchase conditions (see supplemental Fig. 2A, available at www.jneurosci.org as supplemental material). Initially, the pattern vectors of six of the seven runs were used for the training ("training dataset") of the nonlinear support vector machine classifier with a fixed regularization parameter $\mathrm{C}=1$. This provided the basis of the subsequent classification of the pattern vectors of the remaining seventh run ("test dataset") as belonging to either the purchase or no purchase condition (see supplemental Fig. 2B, available at www. jneurosci.org as supplemental material). The procedure was independently repeated seven times with a different run used as the test dataset to achieve a sevenfold cross-validation. (A fivefold cross-validation was realized for two participants in group 2 because complete data were acquired only for 5 of the 7 runs). The amount of purchase-related information of the spatial activation pattern of each spherical cluster was represented by the average decoding accuracy across all cross-validation steps and was assigned to the central voxel $v_{\mathrm{i}}$ of the cluster. Given that the number of considered dimensions (equivalent to number of voxels in a searchlight) exceeded the number of acquired data points, we have to consider that noise was fitted to the training of the classifier. The use of independent data for training and testing, however, controlled for the impact of potential overfitting, and consequently for the overestimation in the prediction accuracy (Vul et al., 2009). Moreover, to control for "peaking" problems, decoding accuracies were calculated by estimating the mean across seven cross-validation steps.

The described classification was successively performed for all clusters created around every measured voxel, resulting in a three-dimensional map of average classification accuracies for each participant. These accuracy maps were then spatially normalized to a standard brain [Montreal Neurological Institute (MNI) EPI template as implemented in SPM2] and resampled to an isotropic spatial resolution of $3 \times 3 \times 3 \mathrm{~mm}^{3}$. Finally, a standard second-level statistical analysis as implemented in SPM2 was performed to identify brain regions that allowed classification of consumer choices across participants in each group. For both groups, analyses were based on the normalized three-dimensional accuracy maps of each subject. Each single point of an individual accuracy map represented the average decoding accuracy of a searchlight surrounding this position across all cross-validation steps. Thus, each value represented the amount of choice-related information of the spatial activation pattern of a surrounding spherical cluster. To assess the statistical significance of these accuracy values across subjects, individual accuracy maps of one group were submitted to a voxelwise one sample $t$ test and contrasted against chance level. Since the classification was based on two alternatives (purchase vs no purchase), chance level was 50\%. Familywise error (FWE) correction for multiple comparisons was implemented to control for false positives. Only regions passing this stringent statistical threshold ( $p<0.05$, FWE-corrected, whole brain) and showing significant decoding accuracies above chance were considered relevant for information encoding (Haynes et al., 2007; Soon et al., 2008).

Supplemental data analysis. After each product presentation, participants in group 1 (high attention) had to rate the attractiveness of the particular car on a four-point scale via a button-press. To ensure that brain regions predictive for subsequent consumer choices do not mainly reflect attractiveness, we conducted an additional multivariate decoding analysis. Except for the GLM parameter estimates representing attractiveness ratings instead of consumer choices, the multivariate searchlight decoding was identical to the one described for the main analysis. Another separate decoding analysis was performed on the button-presses to confirm that regions predicting product choices did not simply encode subsequent motor responses. Here, parameter estimates of the GLM were created based on motor responses indicating subjective attractiveness judgments. Apart from that, the analysis was similar to the one described above to predict consumer choices. In both additional decoding analyses chance level was $25 \%$, because the classification was always based on four alternatives of either attractiveness or button-presses. We considered only regions showing significant decoding accuracies above chance as relevant for encoding of attractiveness and motor responses.

Furthermore, classic univariate analyses were conducted for data from both groups. This enabled us to investigate whether there are single vox- 
els whose mean activation is significantly more strongly activated in one experimental condition of consumer choices compared with another (purchase vs no purchase). The functional imaging data were preprocessed using slice time correction, motion correction, were spatially normalized to a standard stereotaxic space (MNI EPI template), resampled to an isotropic spatial resolution of $3 \times 3 \times 3 \mathrm{~mm}^{3}$ and smoothed with a Gaussian kernel of $6 \mathrm{~mm}$ full-width half-maximum. Except for these differences in the preprocessing, parameter estimates were created as described in the first step of the multivariate decoding approach. Parameter estimates of purchase and no purchase choices were then contrasted against each other on a single-subject level. Subsequently, a second-level statistical analysis was performed to identify regions that were significantly more strongly activated in one of the two conditions across participants.

To test whether engagement of attention in one region of the visual field (corresponding to the square of the fixation task in group 2) can strongly decrease the processing of task-irrelevant background stimuli, we compared the blood oxygenation level-dependent (BOLD) signal change during product exposure of group 2 (low attention) and group 1 (high attention). In the first step, GLM parameter estimates for visual responses during product exposure were created for each participant and contrasted against baseline. Based on these contrasts, the individual BOLD signal change was estimated for each participant. In the second step, these estimates were used to calculate the average BOLD signal change across participants by implementing a second-level analysis for Groups 1 and 2 individually. Subsequently to contrasting the resulting parameter estimates against baseline, we identified the peak activation area of both hemispheres across these two contrast images (located in the left $[\mathrm{MNI}-30,-81,-21]$ and right visual cortex [MNI 27, -84, -18]). Assuming that the visual information was encoded in both hemispheres, we pooled the signal change from both peak activation areas. This was performed for group 1 and group 2 individually. Finally, a $t$ test for independent samples was applied to compare the average signal change in group 1 and group 2.

\section{Results}

\section{Behavioral results}

\section{Consumer choices}

In group 1 (high attention), we obtained functional brain responses while participants closely attended to and actively evaluated products. In group 2 (low attention), participants performed a distraction task at fixation while task-irrelevant products were presented outside the focus of attention on the back of the screen. Subsequent to scanning, participants in both groups had to state their willingness to purchase each particular car. The number of trials assigned to either the purchase or no purchase condition was found to be well balanced within both groups and comparable across them. In group 1, the mean distribution of the declared consumer choices for all products was $54 \%$ no purchase, $5 \%$ "maybe," and $41 \%$ purchase. In group 2, 46\% of the products were chosen to be no purchase trials, $4 \%$ maybe, and $50 \%$ purchase trials across participants. For both groups, profiles of product selection were found to vary across participants (see supplemental Table 1 , available at www.jneurosci.org as supplemental material). Additionally, the results of a $t$ test for independent samples confirmed that product-specific means (group 1: mean $1.87 \pm$ SD 0.83; group 2: mean $1.86 \pm$ SD 0.93$)$ of consumer choices were comparable across both groups $(t=-0.43, p=0.67)$.

\section{Familiarity}

After scanning, participants from both groups were asked whether they had been familiar with the presented products before the experiment. In group 1 (high attention), participants reported the products were familiar in $85 \%$ of all cases and as being unknown in $12 \%$. In group 2 (low attention), participants were acquainted with $87 \%$ of the products before the experiment while $10 \%$ of products were unknown. For the remaining 3\% in both groups the participants stated they were unsure whether they were acquainted with the product before the experiment. For both groups, these findings confirmed that most of the products presented were well known before the experiment.

\section{Task performance and recognition rates}

To examine whether attention was effectively removed from products in the low attention condition as implemented in group 2 , we analyzed the behavioral performance in the visual fixation task during scanning. The high mean percentage of $89 \%$ correct responses indicates that participants attended to the assigned demanding fixation task as instructed. Moreover, we compared the recognition performance in a memory test for both groups (high and low attention) conducted upon completion of the experiment (independent sample of 41 healthy male volunteers: high attention group: $N=20,26.5 \pm 2.8$ years; low attention group: $N=21,25.8 \pm 1.9$ years, mean $\pm \mathrm{SD}$ ). Subsequent to the completion of either the high attention or the low attention condition, participants were presented with single images of 20 cars in randomized order. Ten of these cars were stimuli used in the experiment while the remaining 10 images displayed previously unseen cars. For each product, participants had to state whether this car had been presented during the experiment or not. Size and position of the products on the computer screen were kept constant to facilitate recognition performance. Individual hitrates $[\mathrm{HR}$ (high attention) $=$ mean $0.97 \pm \mathrm{SD} 0.07$, HR(low attention) $=$ mean $0.63 \pm \mathrm{SD} 0.26$ ] as well as false-alarm-rates $[\mathrm{FAR}($ high attention $)=$ mean $0.02 \pm \mathrm{SD} 0.04$, FAR(low attention $)=$ mean $0.22 \pm$ SD 0.18 ] were found to differ significantly between high and low attention conditions (HR: $t=-5.41, p<$ 0.001 ; FAR: $t=4.74, p<0.001)$. Participants in the low attention condition showed lower correct recognition of previously seen cars than participants in the high attention condition. Moreover, they were more likely to incorrectly rate a previously unseen car as having been presented during the experiment. These findings strongly suggest that attention was effectively removed from products in the low attention condition of our experiment.

\section{fMRI results}

Multivariate decoding of subsequent consumer choices

In group 1 (high attention), spatial activation patterns in the prefrontal cortex (PFC), namely in the left medial frontal gyrus (82\% decoding accuracy), the right dorsomedial PFC (75\% decoding accuracy) and the bilateral ventromedial PFC (73\% decoding accuracy) predicted subsequent consumer choices. Moreover, the left insula (73\% decoding accuracy) and the right parahippocampal gyrus ( $72 \%$ decoding accuracy) were found to contain stable information about later product choices (see Table 1 for a complete list of results).

In group 2 (low attention), activation patterns in the left medial PFC (76\% decoding accuracy) and the bilateral insula (right: $82 \%$ decoding accuracy, left: $72 \%$ decoding accuracy) predicted subsequent consumer choices. Neural responses in the left inferior parietal lobe ( $82 \%$ decoding accuracy) and the bilateral superior temporal gyrus (left: 74\% decoding accuracy, right: 70\% decoding accuracy) also encoded choices between cars (see Table 2 for a complete list of results). It should be noted that decoding accuracies in brain regions predicting subsequent consumer choices under high and low attention conditions were found to be comparable (Fig. 2).

To provide further evidence for the statistical validity of the results obtained by this approach, an additional decoding analy- 
Table 1. Brain regions encoding the subsequent consumer choices among actively evaluated products in group 1 (high attention)

\begin{tabular}{|c|c|c|c|c|c|c|c|c|}
\hline \multirow[b]{2}{*}{ Brain region } & \multirow[b]{2}{*}{ Side } & \multirow[b]{2}{*}{ BA } & \multicolumn{2}{|c|}{ Accuracy } & \multirow[b]{2}{*}{$t$ value } & \multicolumn{3}{|c|}{ MNI coordinates } \\
\hline & & & M & SE & & $x$ & $y$ & $z$ \\
\hline \multicolumn{9}{|l|}{ Frontal lobe } \\
\hline Medial frontal gyrus & $\mathrm{L}$ & 9 & 82 & 3.3 & 9.49 & -21 & 27 & 30 \\
\hline mPFC (dorsal) & $\mathrm{R}$ & 10 & 75 & 2.6 & 9.53 & 9 & 57 & 21 \\
\hline mPFC (ventral) & $\mathrm{L}$ & 10 & 73 & 2.3 & 9.96 & -9 & 63 & 12 \\
\hline \multicolumn{9}{|l|}{ Limbic lobe } \\
\hline Insula & $\mathrm{L}$ & & 73 & 2.3 & 9.80 & -36 & -6 & 0 \\
\hline Parahippocampal gyrus & $\mathrm{R}$ & 30 & 72 & 1.9 & 10.98 & 30 & -57 & 3 \\
\hline \multicolumn{9}{|l|}{ Occipital lobe } \\
\hline \multirow[t]{2}{*}{ Inferior occipital lobe } & $\mathrm{L}$ & 18 & 73 & 2.2 & 10.44 & -15 & -105 & -6 \\
\hline & $\mathrm{L}$ & 18 & 70 & 2.0 & 9.96 & -27 & -93 & -15 \\
\hline Cerebellum & $\mathrm{R}$ & & 77 & 2.7 & 9.69 & 9 & -66 & -36 \\
\hline
\end{tabular}

Results are reported on a statistical level of $p<0.05$, FWE-corrected; only peak activations of clusters are listed. L, Left hemisphere; R, right hemisphere; $M$, mean; BA, Brodmann area.

Table 2. Brain regions encoding the subsequent consumer choices among passively presented, task-irrelevant products in group 2 (low attention)

\begin{tabular}{|c|c|c|c|c|c|c|c|c|}
\hline \multirow[b]{2}{*}{ Brain region } & \multirow[b]{2}{*}{ Side } & \multirow[b]{2}{*}{$B A$} & \multicolumn{2}{|c|}{ Accuracy } & \multirow[b]{2}{*}{$t$ value } & \multicolumn{3}{|c|}{ MNI coordinates } \\
\hline & & & M & SE & & $x$ & $y$ & $Z$ \\
\hline \multicolumn{9}{|l|}{ Frontal lobe } \\
\hline \multirow[t]{3}{*}{ Middle frontal gyrus } & $\mathrm{L}$ & 8 & 79 & 2.8 & 10.45 & -24 & 36 & 42 \\
\hline & $\mathrm{L}$ & 8 & 76 & 1.8 & 10.70 & -21 & 36 & 51 \\
\hline & $\mathrm{L}$ & 8 & 72 & 1.9 & 11.35 & -39 & 33 & 42 \\
\hline \multirow[t]{2}{*}{$\mathrm{mPFC}$} & $\mathrm{L}$ & 10 & 77 & 2.2 & 12.38 & -15 & 57 & 24 \\
\hline & $\mathrm{L}$ & 10 & 76 & 2.0 & 12.83 & -6 & 51 & 18 \\
\hline $\mathrm{mOFC}$ & $\mathrm{L}$ & 11 & 74 & 2.0 & 12.20 & -12 & 42 & -18 \\
\hline Inferior frontal gyrus & $\mathrm{L}$ & 45 & 73 & 2.1 & 10.90 & -51 & 24 & 15 \\
\hline \multicolumn{9}{|l|}{ Limbic lobe } \\
\hline \multirow[t]{2}{*}{ Insula } & $\mathrm{R}$ & & 82 & 2.6 & 5.19 & 39 & -3 & 15 \\
\hline & $\mathrm{L}$ & & 72 & 1.8 & 12.46 & -42 & 15 & -6 \\
\hline \multicolumn{9}{|l|}{ Parietal lobe } \\
\hline \multirow[t]{2}{*}{ Inferior parietal lobe } & $\mathrm{L}$ & 40 & 82 & 2.7 & 11.81 & -57 & -36 & 27 \\
\hline & $\mathrm{R}$ & 40 & 73 & 2.1 & 10.66 & 60 & -39 & 30 \\
\hline \multicolumn{9}{|l|}{ Temporal lobe } \\
\hline \multirow[t]{3}{*}{ Superior temporal gyrus } & $\mathrm{L}$ & 38 & 74 & 1.8 & 13.31 & -51 & 12 & -18 \\
\hline & $\mathrm{R}$ & 42 & 75 & 1.8 & 13.40 & 54 & -33 & 15 \\
\hline & $\mathrm{R}$ & 42 & 70 & 1.5 & 13.62 & 63 & -36 & 18 \\
\hline
\end{tabular}

Results are reported on a statistical level of $p<0.05$, FWE-corrected; only peak activations of clusters are listed. L, Left hemisphere; $R$, right hemisphere; $M$, mean; BA, Brodmann area; OFC, orbitofrontal cortex.

sis was conducted for data of both groups. When test datasets were randomly assigned to either the purchase or no purchase condition during the testing phase of the classifier, no statistical significant prediction of consumer choices could be achieved (FDR and FWE-corrected). This was true for data from both groups independently. Moreover, decoding accuracies in brain regions that were informative in the original analysis were at chance level (50\%) when the test data were randomly allocated to the conditions (see supplemental Fig. 3, available at www. jneurosci.org as supplemental material). This finding speaks against potential methodological concerns such as possible biases inherent in the testing procedure and insufficient corrections for multiple comparisons. Finally, we investigated whether a combination of two informative classifiers [i.e., medial prefrontal cortex (mPFC) and insula] could improve the overall prediction accuracy. Compared with decoding results of single searchlights in these areas, the weighted classification using decision values of the first-level decoding enhanced the prediction by $7 \%$ and by $5 \%$ respectively.

Neural activation in the ventral striatum has frequently been implicated in financial decision-making, preference-related processing of products as well as purchases (Erk et al., 2002; Kuhnen and Knutson, 2005; Knutson and Bossaerts, 2007; Knutson et al., 2007; Schaefer and Rotte, 2007a). To investigate whether spatial activation patterns in these regions would be found to contain stable information about product choices when smaller searchlights (radius of 2 voxels) were used, an additional decoding analysis was performed. At a more liberal statistical threshold of $p<0.00001$ (uncorrected), this analysis revealed predictive information in the striatum (see supplemental Fig. 4, available at www.jneurosci.org as supplemental material).

Univariate comparisons of subsequent consumer choices

In both groups, classic univariate comparisons did not reveal any activation differences between products that participants were willing to purchase and those they were not. This strongly suggests that multivariate pattern classification is capable of extracting information which conventional analyses fail to detect.

\section{Multivariate decoding of attractiveness judgments}

Participants in group 1 were instructed to judge the subjective attractiveness of a particular car after each product presentation. Spatial activation patterns in the right middle frontal gyrus $(47 \%$ decoding accuracy, [MNI 30, 12, 33]), medial frontal gyrus (43\% decoding accuracy, [MNI 15, 33, 45]) and left orbitofrontal cortex $(40 \%$ decoding accuracy, $[\mathrm{MNI}-27,33,18])$ were found to encode attractiveness judgments during product presentation. Activity in the left (51\% decoding accuracy, [MNI - 15, 24, 30]) and right dorsal anterior cingulate cortex (49\% decoding accuracy, [MNI 15, 24, 30]), left (41\% decoding accuracy, [MNI - 18, 


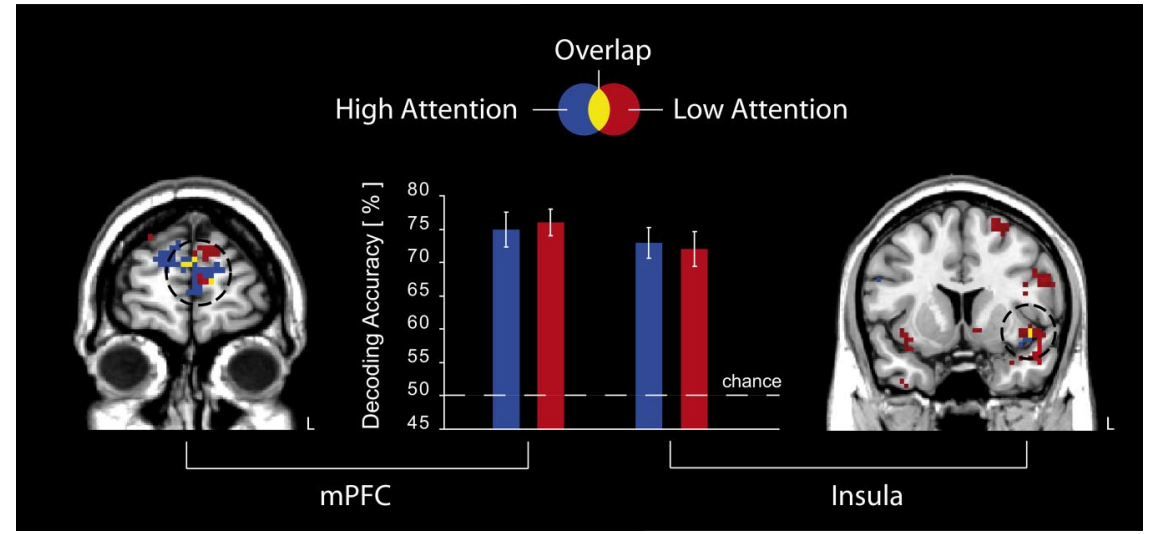

Figure 2. Brain regions encoding subsequent consumer choices in both groups. Multivariate searchlight decoding (radius of 4 voxels) was applied to functional brain responses obtained during product exposure to predict subsequent consumer choices (chance level 50\%). Spatial activation patterns in the mPFC and the insula were found to encode these choices when participants did not explicitly deliberate on purchases ( $p<0.05$, FWE-corrected). Importantly, this applied to situations when participants closely attended to and actively evaluated products ("high attention" group 1, blue) as well as when products were passively presented outside the focus of attention ("low attention" group 2, red). The amount of predictive information in the brain responses was found to be comparably high in both groups. The graph displays mean decoding accuracies and SEs across participants for both regions and both groups. For illustrative purposes, the contrasts are shown at $p<0.0001$ (uncorrected) with a cluster threshold of 10 voxels (L indicates left hemisphere).

$-45,12])$ and right posterior cingulate cortex (42\% decoding accuracy, [MNI 9, - 42, 12]) and left insula (40\% decoding accuracy, $[\mathrm{MNI}-42,9,-3]$ ) were also found to be predictive of evaluation scores $(p<0.05$, FWE-corrected, chance level 25\% for attractiveness ratings 1-4). For a complete list of results, see supplemental Table 3, available at www.jneurosci.org as supplemental material. Importantly, no direct overlap of regions predicting consumer choices and those predicting attractiveness scores could be observed (see supplemental Fig. 5, available at www.jneurosci.org as supplemental material). This finding strongly supports the notion that the information encoding consumer choices is not merely based on evaluative judgments of attractiveness.

\section{Multivariate decoding of button presses}

To ensure that the neural activity during the product viewing phase was not confounded by the preparation of the motor response, we applied a randomized response-button mapping. This mapping scheme was only presented after the removal of the product to isolate the motor response from the processing of product information. As expected, we found no brain region that was informative about the subsequent motor response during the product-viewing phase. This finding strongly suggests that the prediction of subsequent consumer choices was not based on motor preparation during product exposure. During the presentation of the response-mapping scheme (subsequent to the product presentation), the bilateral motor cortex ([MNI 48, -21, 57] and $[\mathrm{MNI}-42,-21,54])$ predicted the current button-press ( $p<0.05$, FWE-corrected, chance level 25\% for buttons 1-4). Importantly, no neural structure predictive of subsequent consumer choices contained information about the associated motor responses (see supplemental Fig. 6, available at www.jneurosci. org as supplemental material). This finding indicates that the prediction of consumer choices was not related to neural processes involved in the execution of motor responses.

Attention modulation in the visual cortex

Engagement of attention in one region of the visual field (corresponding to the square of the fixation task) has been suggested to decrease the visual processing of taskirrelevant background stimuli (cars). To test this assumption, we compared the BOLD signal change in the visual cortex during product exposure in the high and low attention conditions. Consistent with our hypothesis, we found a significant difference in the BOLD signal change evoked by the presentation of cars in the high attention and low attention group $(t$ test for independent samples, $t=5.15, p<$ $0.001)$. More precisely, the results demonstrated that the responses in the visual cortex evoked by the task-irrelevant images of cars which were presented outside the focus of attention were reduced compared with when the images are actively evaluated and attended to. This finding indicates that the engagement of attention in one region of the visual field (corresponding to the square of the fixation task) decreased the processing of taskirrelevant products in the background.

\section{Discussion}

The present study investigated the impact of product-related attention on neural predictors of consumer choices. Activation patterns in the insula and the mPFC were found to predict these choices under high and low attention processing (Fig. 2). Thus, a close match of predictive brain regions was revealed independent of spatial attention to products. This demonstrates that processing of unattended stimuli can proceed beyond object processing (Peelen et al., 2009) to a stage that even allows the prediction of consumer choices. Importantly, the amount of predictive information in these areas was comparably as high when task-irrelevant products were presented outside the focus of attention as when they were actively evaluated and attended to. Congruent with the notion of unconscious environmental triggers for automatic processes in consumer settings (Chartrand, 2005), this indicates that a prediction of economic choices from brain responses does not necessarily depend on attentive processing of products.

Notably, these findings were achieved with participants who were naive about the necessity of a subsequent choice. This is in line with behavioral findings reporting that consumer-related goals can automatically be activated, guiding subsequent consumer behavior and choices even outside of conscious awareness (Bargh, 2002; Chartrand, 2005; Dijksterhuis et al., 2005). Moreover, it is consistent with previous data showing that brain responses reflect preference choices when participants evaluate stimuli with respect to other, non-preference-related aspects (Kim et al., 2007; Lebreton et al., 2009). The current study goes beyond these results by demonstrating that brain responses predict subsequent choices even in the absence of spatial attention to choice options.

Neural activation in the insula and the mPFC has been shown to predict consumer choices when participants closely attend to products and explicitly deliberate about purchases (Knutson et al., 2007). Given that the prediction of consumer choices in the present study was achieved in the absence of explicit deliberation and without a priori assumptions about informative regions, this further supports the role of both areas in economic decisionmaking. Spatial activation patterns in the insula and the mPFC 
were also reported to predict behavioral choices in the following trial of a reward-based decision-making task (Hampton and O'Doherty, 2007). In line with this finding, both structures have been suggested to underlie the neural representation of the expected reward value (Knutson et al., 2005; Preuschoff et al., 2006; Hampton et al., 2007; Hare et al., 2008; Rolls et al., 2008), a concept central to microeconomic and psychological models of decision-making (Knutson and Bossaerts, 2007; Loewenstein et al., 2008; Rangel et al., 2008). Cultural stimuli such as cars and logos of car manufacturers can signal potential social dominance or wealth and have been shown to modulate activity in the reward circuit (Erk et al., 2002; Schaefer and Rotte, 2007a). It can be assumed that products in the present experiment differed in their expected reward value depending on whether they were subsequently chosen to be purchased or not (Sharot et al., 2009). We suggest that distinguishable activation patterns in the insula and the $\mathrm{mPFC}$ reflected these different reward values and-even without full attention - contributed to subsequent hypothetical consumer choices.

Activation in the $\mathrm{mPFC}$ - particularly the ventral part-has also been reported to reflect product-related preference and attractiveness judgments (Paulus et al., 2003; McClure et al., 2004; Deppe et al., 2005; Plassmann et al., 2008; Lebreton et al., 2009; Luu and Chau, 2009). The dorsal mPFC, on the other hand, has been proposed to be involved in the processing of brand knowledge (Schaefer et al., 2006; Schaefer and Rotte, 2007b), the impact of which on consumer preferences and choices is well known (McClure et al., 2004; Lee et al., 2006). Considering this evidence, it is likely that the predictive information encoded in the mPFC might have been influenced by subjective valuation and the brand and price information.

It can be assumed that global product valuation was a major source for subsequent choices. This is particularly likely, given that no further product-related information was provided during the experiment to avoid drawing participants' attention to the potential choice options. Therefore, it could be speculated that the predictive information merely reflects global attractiveness or desirability of products. However, many cognitive factors as well as their interactions with automatic valuation processes might contribute to a complex choice such as those for a new car. This is particularly likely for participants who stated an interest in cars and are assumed to possess relevant knowledge on the items. Consistent with this notion product choices were not entirely determined by attractiveness judgments. Thus, only partial correlations between product-specific attractiveness ratings and consumer choices were found for both groups in the present experiment (see supplemental Table 2, available at www. jneurosci.org as supplemental material). Additionally, no direct overlap of brain regions predicting attractiveness judgments and those being informative about consumer choices could be identified (see supplemental Table 3 and supplemental Fig. 5, available at www.jneurosci.org as supplemental material). Together, these findings indicate that the prediction of product choices was not mainly due to global evaluations of attractiveness but might reflect automatic choice-related processing itself. This is in line with previous results showing that brain activation reflecting subsequent preference decisions were not merely responding to attractiveness of stimuli (Lebreton et al., 2009). However, more research is needed to specifically examine choice-relevant dimensions with the current paradigms, possibly testing explicit models of multiattribute decision-making (Dijksterhuis et al., 2006; Lassiter et al., 2009). Given that consumer choices for each product were found to vary from participant to participant, it is also unlikely that the predictive information was based on physical properties of single cars (see supplemental Table 1, available at www.jneurosci.org as supplemental material). Results of an additional decoding analysis also demonstrate that the prediction of consumer choices was not related to neural processes involved in the preparation of motor responses (see supplemental Fig. 6, available at www. jneurosci.org as supplemental material).

Another point that needs to be addressed concerns the question of whether attention was effectively attenuated by engaging in the distraction task in group 2. Evidence of successful distraction comes from differences in memory performance, obtained for both groups after the experiment. Recognition rates of cars were strongly decreased in participants who performed the distraction task compared with participants who actively evaluated products. Moreover, neural responses in the visual cortex evoked by task-irrelevant, unattended images of cars were found to be reduced compared with images that were actively evaluated and fully attended to. This is consistent with previous research demonstrating that the engagement of attention in one region of the visual field strongly decreases the processing of irrelevant background stimuli (Rees et al., 1997). It can therefore be considered that the attention to products in group 2 was strongly diminished compared with group 1 . As in most attention studies, there is a possibility of weak but residual attention to unattended target stimuli. However, our key finding is that a strong reduction in attention does not affect the choice-predictive information. Purchase-related information encoded in the brain was not reduced, but was comparably high in low and high attention conditions. Furthermore, we found a close match of predictive brain regions during high and low attentional processing of products. Together, this indicates that choice-related processing does not necessarily require close attention to products and can even occur in cases where sensory signal processing is attenuated due to a removal of attention. Although it remains possible that the choice was not explicitly calculated during product presentation, the high predictive accuracy reveals that choice-related processes have already reached a high level of completion.

The present study implemented hypothetical choices, which are commonly applied in marketing research. Nevertheless, it needs to be explored whether the same activation patterns are predictive for actual purchases- beyond the stated willingness to buy. This would allow checking for potential biases such as the tendency to overstate the willingness to pay or vote in case of hypothetical choices (List and Gallet, 2001; Murphy et al., 2005). Requiring expenses would likely involve losses and more relative comparisons between available products, including changes in the reference point for product valuation (FitzGerald et al., 2009; Sharot et al., 2009). Real purchases might also engage processes of perceived justification, anticipated regret, time pressure or self esteem (Plassmann et al., 2007). Finally, it might be that making actual purchases differs from our experimental setting in terms of the strategies used for information acquisition and information integration across multiple relevant dimensions. However, it should be noted that informative brain regions as identified with the present approach are strikingly consistent with previous findings that implemented actual purchases (Knutson et al., 2007). Further research might also address the generalizability of our findings to other product categories, including different goods (e.g., cars vs coffee) and different types of purchases (e.g., routine vs new), as well as to people who are not interested in the product group.

In summary, we found a close match of brain regions predicting consumer choices for both high and low attentional processing of products. Importantly, the amount of predictive information was 
found to remain persistently high when task-irrelevant products were presented outside the focus of attention. Altogether, these findings support the notion that even complex and important economic choices can be prepared automatically, in the absence of explicit deliberation and without attention to products.

\section{References}

Bargh JA (2002) Losing consciousness: automatic influences on consumer judgment, behavior, and motivation. J Consum Res 29:280-285.

Chartrand TL (2005) The role of conscious awareness in consumer behavior. J Consum Psychol 15:203-210.

Cox DD, Savoy RL (2003) Functional magnetic resonance imaging (fMRI) "brain reading": detecting and classifying distributed patterns of fMRI activity in human visual cortex. Neuroimage 19:261-270.

Deppe M, Schwindt W, Kugel H, Plassmann H, Kenning P (2005) Nonlinear responses within the medial prefrontal cortex reveal when specific implicit information influences economic decision making. J Neuroimaging 15:171-182.

Dijksterhuis A, Smith PK, van Baaren RB, Wigboldus DHJ (2005) The unconscious consumer: effects of environment on consumer behavior. J Consum Psychol 15:193-202.

Dijksterhuis A, Bos MW, Nordgren LF, van Baaren RB (2006) On making the right choice: the deliberation-without-attention effect. Science 311: 1005-1007.

Erk S, Spitzer M, Wunderlich AP, Galley L, Walter H (2002) Cultural objects modulate reward circuitry. Neuroreport 13:2499-2503.

FitzGerald THB, Seymour B, Dolan RJ (2009) The role of human orbitofrontal cortex in value comparison for incommensurable objects. J Neurosci 29:8388-8395.

Friston KJ, Holmes AP, Worsley KJ, Poline JP, Frith CD, Frackowiak RSJ (1994) Statistical parametric maps in functional imaging: a general linear approach. Hum Brain Mapp 2:189-210.

Hampton AN, O'Doherty JP (2007) Decoding the neural substrates of reward-related decision making with functional MRI. Proc Natl Acad Sci U S A 104:1377-1382.

Hampton AN, Adolphs R, Tyszka MJ, O’Doherty JP (2007) Contributions of the amygdala to reward expectancy and choice signals in human prefrontal cortex. Neuron 55:545-555.

Hare TA, O'Doherty J, Camerer CF, Schultz W, Rangel A (2008) Dissociating the role of the orbitofrontal cortex and the striatum in the computation of goal values and prediction errors. J Neurosci 28:5623-5630.

Haxby JV, Gobbini MI, Furey ML, Ishai A, Schouten JL, Pietrini P (2001) Distributed and overlapping representations of faces and objects in ventral temporal cortex. Science 293:2425-2430.

Haynes JD, Rees G (2006) Decoding mental states from brain activity in humans. Nat Rev Neurosci 7:523-534.

Haynes JD, Sakai K, Rees G, Gilbert S, Frith C, Passingham RE (2007) Reading hidden intentions in the human brain. Curr Biol 17:323-328.

Kamitani Y, Tong F (2005) Decoding the visual and subjective contents of the human brain. Nat Neurosci 8:679-685.

Kastner S, Ungerleider LG (2000) Mechanisms of visual attention in the human cortex. Annu Rev Neurosci 23:315-341.

Kim H, Adolphs R, O’Doherty JP, Shimojo S (2007) Temporal isolation of neural processes underlying face preference decisions. Proc Natl Acad Sci U S A 104:18253-18258.

Knutson B, Bossaerts P (2007) Neural antecedents of financial decisions. J Neurosci 27:8174-8177.

Knutson B, Taylor J, Kaufman M, Peterson R, Glover G (2005) Distributed neural representation of expected value. J Neurosci 25:4806-4812.

Knutson B, Rick S, Wimmer GE, Prelec D, Loewenstein G (2007) Neural predictors of purchases. Neuron 53:147-156.

Kriegeskorte N, Goebel R, Bandettini P (2006) Information-based functional brain mapping. Proc Natl Acad Sci U S A 103:3863-3868.

Kriegeskorte N, Simmons WK, Bellgowan PSF, Baker CI (2009) Circular analysis in systems neuroscience: the dangers of double dipping. Nat Neurosci 12:535-540.

Kuhnen CM, Knutson B (2005) The neural basis of financial risk taking. Neuron 47:763-770.

Lassiter GD, Lindberg MJ, González-Vallejo C, Bellezza FS, Phillips ND
(2009) The deliberation-without-attention effect: evidence for an artifactual interpretation. Psychol Sci 20:671-675.

Lebreton M, Jorge S, Michel V, Thirion B, Pessiglione M (2009) An automatic valuation system in the human brain: evidence from functional neuroimaging. Neuron 64:431-439.

Lee L, Frederick S, Ariely D (2006) Try it, you'll like it: the influence of expectation, consumption, and revelation on preferences for beer. Psychol Sci 17:1054-1058.

List JA, Gallet CA (2001) What experimental protocol influence disparities between actual and hypothetical stated values. Environ Res Econ 20:241254.

Loewenstein G, Rick S, Cohen JD (2008) Neuroeconomics. Annu Rev Psychol 59:647-672.

Luu S, Chau T (2009) Decoding subjective preference from single-trial near-infrared spectroscopy signals. J Neural Eng 6:016003.

Martínez A, Anllo-Vento L, Sereno MI, Frank LR, Buxton RB, Dubowitz DJ, Wong EC, Hinrichs H, Heinze HJ, Hillyard SA (1999) Involvement of striate and extrastriate visual cortical areas in spatial attention. Nat Neurosci 2:364-369.

McClure SM, Li J, Tomlin D, Cypert KS, Montague LM, Montague PR (2004) Neural correlates of behavioral preference for culturally familiar drinks. Neuron 44:379-387.

Mitchell TM, Hutchinson R, Niculescu RS, Pereira F, Wang X, Just M, Newman $S$ (2004) Learning to decode cognitive states from brain images. Machine Learn 57:145-175.

Murphy J, Allen PG, Stevens TH, Weatherhead D (2005) A meta-analysis of hypothetical bias in stated preference valuation. Environ Res Econ 30: 313-325.

Norman KA, Polyn SM, Detre GJ, Haxby JV (2006) Beyond mind-reading: multi-voxel pattern analysis of fMRI data. Trends Cogn Sci 10:424-430.

O'Doherty J, Winston J, Critchley H, Perrett D, Burt DM, Dolan RJ (2003) Beauty in a smile: the role of medial orbitofrontal cortex in facial attractiveness. Neuropsychologia 41:147-155.

Paulus MP, Rogalsky C, Simmons A, Feinstein JS, Stein MB (2003) Increased activation in the right insula during risk-taking decision making is related to harm avoidance and neuroticism. Neuroimage 19:1439-1448.

Peelen MV, Li FF, Kastner S (2009) Neural mechanisms of rapid natural scene categorization in human visual cortex. Nature 460:94-97.

Plassmann H, Ambler T, Brautigam S, Kenning P (2007) What can advertisers learn from neuroscience? Int J Advertising 26:151-175.

Plassmann H, O’Doherty J, Shiv B, Rangel A (2008) Marketing actions can modulate neural representations of experienced pleasantness. Proc Natl Acad Sci U S A 105:1050-1054.

Preuschoff K, Bossaerts P, Quartz SR (2006) Neural differentiation of expected reward and risk in human subcortical structures. Neuron 51:381-390.

Rangel A, Camerer C, Montague PR (2008) A framework for studying the neurobiology of value-based decision making. Nat Rev Neurosci 9:545-556.

Rees G, Frith CD, Lavie N (1997) Modulating irrelevant motion perception by varying attentional load in an unrelated task. Science 278:1616-1619.

Rolls ET, McCabe C, Redoute J (2008) Expected value, reward outcome, and temporal difference error representations in a probabilistic decision task. Cereb Cortex 18:652-663.

Schaefer M, Rotte M (2007a) Favorite brands as cultural objects modulate reward circuit. Neuroreport 18:141-145.

Schaefer M, Rotte M (2007b) Thinking on luxury or pragmatic brand products: brain responses to different categories of culturally based brands. Brain Res 1165:98-104.

Schaefer M, Berens H, Heinze HJ, Rotte M (2006) Neural correlates of culturally familiar brands of car manufacturers. Neuroimage 31:861-865.

Sharot T, De Martino B, Dolan RJ (2009) How choice reveals and shapes expected hedonic outcome. J Neurosci 29:3760-3765.

Soon CS, Brass M, Heinze HJ, Haynes JD (2008) Unconscious determinants of free decisions in the human brain. Nat Neurosci 11:543-545.

Vul E, Harris C, Winkielman P, Pashler H (2009) Puzzlingly high correlations in fMRI studies of emotion, personality, and social cognition. Perspect Psychol Sci 4:274-290. 